ARTICLE

Received 9 Apr 2015 | Accepted 13 Aug 2015 | Published 22 Sep 2015

\title{
Circularly polarized light detection with hot electrons in chiral plasmonic metamaterials
}

Wei Li ${ }^{1}$, Zachary J. Coppens ${ }^{1}$, Lucas V. Besteiro ${ }^{2}$, Wenyi Wang ${ }^{3}$, Alexander O. Govorov ${ }^{2}$ \& Jason Valentine ${ }^{1}$

Circularly polarized light is utilized in various optical techniques and devices. However, using conventional optical systems to generate, analyse and detect circularly polarized light involves multiple optical elements, making it challenging to realize miniature and integrated devices. While a number of ultracompact optical elements for manipulating circularly polarized light have recently been demonstrated, the development of an efficient and highly selective circularly polarized light photodetector remains challenging. Here we report on an ultracompact circularly polarized light detector that combines large engineered chirality, realized using chiral plasmonic metamaterials, with hot electron injection. We demonstrate the detector's ability to distinguish between left and right hand circularly polarized light without the use of additional optical elements. Implementation of this photodetector could lead to enhanced security in fibre and free-space communication, as well as emission, imaging and sensing applications for circularly polarized light using a highly integrated photonic platform.

\footnotetext{
${ }^{1}$ Department of Mechanical Engineering, Vanderbilt University, Nashville, Tennessee 37212, USA. ${ }^{2}$ Department of Physics and Astronomy, Ohio University, Athens, Ohio 45701, USA. ${ }^{3}$ Department of Electrical Engineering, Vanderbilt University, Nashville, Tennessee 37212, USA. Correspondence and requests for materials should be addressed to J.V. (email: jason.g.valentine@vanderbilt.edu).
} 
C ircularly polarized light (CPL) is utilized in various optical techniques and devices, ranging from quantum computation $^{1-3}$ and spin optical communication ${ }^{4}$ to circular dichroism (CD) spectroscopy ${ }^{5}$ and magnetic recording ${ }^{6}$. In $\mathrm{CPL}$, the electric field vector travels along a helical trajectory, either clockwise or counterclockwise ${ }^{7}$, and it can be generated from two linearly polarized light (LPL) waves with perpendicular electric field vectors that are oscillating with a $90^{\circ}$ phase shift. Distinguishing between the two polarizations of $\mathrm{CPL}$ is inherently difficult with conventional photodetectors due to the fact that conventional semiconductors lack intrinsic chirality. However, in nature, some species of mantis shrimp can detect CPL and use it as a private channel of communication that is unavailable to both predators and potential competitors ${ }^{8}$. The origin of this ability comes from specialized cells in the retina that serve as quarter-wave plates that sit atop LPL sensitive photoreceptors and convert CPL into $\mathrm{LPL}^{8}$. This is done in the same manner as CPL is detected using conventional optics, namely, through the combination of a non-chiral photodetector with a quarter waveplate and a linear polarizer. While ultracompact optical elements and devices including CPL sources $^{9,10}$, quarter waveplates ${ }^{11}$, polarizers ${ }^{12}$ and beam splitters $^{13,14}$ have been successfully demonstrated, the use of multiple optical elements to distinguish CPL makes it challenging to realize miniature and integrated CPL detectors.

One alternative technique is the use of chiral media as the active material in a photodetector. A chiral medium, one in which the unit cell cannot be superimposed on its mirror image, responds differently for left-handed circularly polarized (LCP) light and right-handed circularly polarized (RCP) light. Chiral media exists in nature, for example, the iridescent metallic green beetle, Chrysina gloriosa, selectively reflects LCP light but absorbs RCP due to structural chirality ${ }^{15}$, while sugar molecules, acids and proteins exhibit intrinsic chirality. Recently, a chiral organic semiconductor transistor has been demonstrated for direct detection of CPL using the intrinsic chiral response of helicene at a wavelength of $365 \mathrm{~nm}$ (ref. 16). Although this technique shows promise, the organic semiconductor is unstable in ambient conditions. It also has a limited response time and the operational wavelength is limited to the ultraviolet regime.

Over the past decade, advances in plasmonics have provided a mechanism for strong light-matter interaction leading to strong field enhancement and resonant scattering and absorption. Furthermore, surface plasmon resonances offer new insights into creating artificial media, or metamaterials, with interesting optical properties such as strong chirality ${ }^{17,18}$ that is several orders of magnitude larger than that of chiral molecules. A number of chiral metamaterials based on plasmonic building blocks including spiral $^{19}$, fish-scale metamaterials ${ }^{20}$, helix ${ }^{12,21,22}$, oligomers $^{23}$ and twisted metamaterials ${ }^{24,25}$ ' have been demonstrated to date using both bottom-up ${ }^{21,26}$ and top-down approaches ${ }^{12,23-25}$. In addition, Archimedes spiral designs have been utilized to enable chiral-selective field enhancements in semiconductors, leading to selective photocurrent for LCP and RCP light, with an experimentally measured ratio of 1.13 (ref. 19). The use of plasmonic elements also opens the door to photon energy harvesting through hot carrier generation and injection $^{27,28}$, leading to a new scheme for photodetection ${ }^{29-35}$ and photocatalysis ${ }^{36,37}$. Hot carriers generated from the non-radiative decay of surface plasmons can be captured with electron acceptors such as metal-semiconductor Schottky interfaces $^{29-32,35,38}$ or metal-insulator-metal junctions ${ }^{33,34}$, resulting in photocurrent. This scheme enables direct conversion of light into an electrical signal-based solely on the photoexcited carriers generated from the absorption in plasmonic nanostructures. The tunability of the plasmonic structures enables a straightforward tailoring of the response of a photodetector including the working wavelength, bandwidth and polarization dependence $29,32,34,35$.

In the present work, we propose and experimentally demonstrate an ultracompact CPL detector in which the ability to distinguish LCP and RCP comes from the engineered chirality in plasmonic nanostructures. Photodetection is based on hot electron injection into silicon with operation in the telecommunications band. Implementation of this CPL photodetector could lead to enhanced security in fibre and free-space communication as well as emission, imaging and sensing applications for CPL.

\section{Results}

Chiral metamaterial and device design. Our proposed chiral metamaterial (Fig. 1a) is a periodic array of chiral metamolecules, with a unit cell consisting of a ' $\mathrm{Z}$ '-shaped silver (Ag) antenna on top of a poly(methyl methacrylate) (PMMA) spacer a

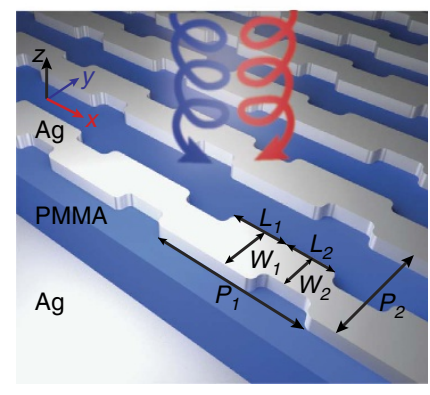

b

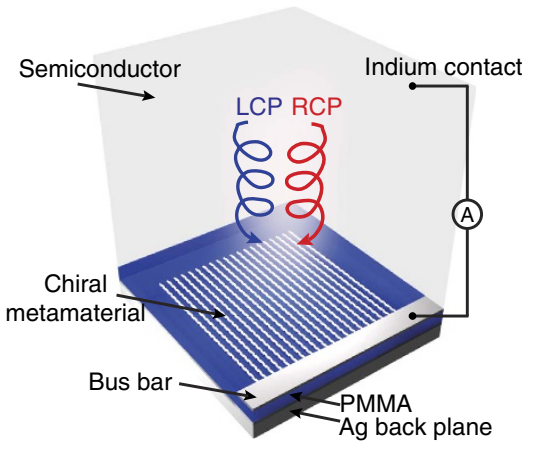

C Ohmic contact: indium

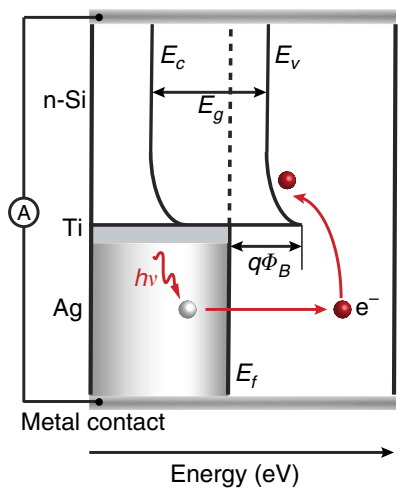

Figure 1 | Schematic of the chiral metamaterial and the CPL detector. (a) Schematic of the chiral metamaterial consisting of the chiral plasmonic meta-molecule array, dielectric spacer and metal backplane. The dimensions of the meta-molecules are $L_{1}=125 \mathrm{~nm}, L_{2}=105 \mathrm{~nm}, W_{1}=115 \mathrm{~nm}$, $W_{2}=85 \mathrm{~nm}, P_{1}=335 \mathrm{~nm}$ and $P_{2}=235 \mathrm{~nm}$. The thickness of the meta-molecules, dielectric spacer and the metal backplane are 40,160 and $100 \mathrm{~nm}$, respectively. (b) Schematic of the CPL detector consisting of a chiral metamaterial integrated with a semiconductor that serves as a hot electron acceptor. The Ohmic contact on $\mathrm{Si}$ is formed by soldering indium. The circuit is formed by wire bonding to the silver bus bar and indium. (c) Energy band diagram of the CPL detector. A Schottky barrier is formed between Si and the Ti interfacial layer. The hot electrons that are photo-generated in the Ag metamaterial are then injected over this barrier into the Si. 
and an optically thick Ag backplane. The unit cell shape of this chiral metamaterial allows for an inherent electrical connection among all the elements, forming nanowires, while a silver bus bar is used to electrically connect the nanowires (Fig. 1b). The whole device is realized by placing an n-type silicon wafer in contact with the antenna layer, forming a Schottky barrier (Fig. 1c). Light is incident on the frontside of the $\mathrm{Si}$ wafer, transmitting to the backside where the chiral metamaterial absorbs photons of a particular handedness, generating electrons within the metal at higher energy states. The energetic electrons (or hot electrons) with energy higher than the Schottky barrier can emit over the Schottky interface, leading to a detectable current (Fig. 1c).

To investigate the optical response of the device on CPL illumination, full-wave electromagnetic simulations were first performed. LCP and RCP light are incident onto the frontside of the silicon wafer, transmitting to the backside where the chiral metamaterial selectively absorbs light with a particular handedness while reflecting the other. For example, a left-handed (LH) chiral metamaterial acts as a perfect LCP light absorber at the resonant wavelength while reflecting nearly $90 \%$ of the RCP light (Fig. 2a) and vice versa for the right-handed (RH) chiral metamaterial (Fig. $2 \mathrm{~b})$, leading to a $\mathrm{CD}\left(\mathrm{CD}=A_{\mathrm{LCP}}-A_{\mathrm{RCP}}\right)$ of almost 0.9 (Fig. 2c). Replacing the antenna layer with an unstructured Ag film results in 95\% reflection, indicating we are nearly at the Drude damping limit. The chiral response can also be tuned across the entire telecommunication regime as shown in Fig. 2d. The minimum and maximum working wavelengths are only limited by the semiconductor bandgap energy and the Schottky barrier height, respectively. The limit imposed by silicon's bandgap is due to the fact that we are illuminating the device through the silicon wafer and must avoid direct absorption by the semiconductor.

To understand the origin of the strong $\mathrm{CD}$, the CPL electric field vector can be decomposed as two orthogonal LPL electric field vectors, $E_{x}$ and $E_{y}$, that are oscillating with a $90^{\circ}$ phase shift:

$$
\begin{gathered}
E_{\mathrm{LCP}}=\left(E_{x}, E_{y}\right)^{T}=\left(\frac{1}{\sqrt{2}}, \exp \left(-\frac{\pi}{2} i\right)\right)^{T} \\
E_{\mathrm{RCP}}=\left(E_{x}, E_{y}\right)^{T}=\left(\frac{1}{\sqrt{2}}, \exp \left(\frac{\pi}{2} i\right)\right)^{T}
\end{gathered}
$$

These fields are input into a multiple reflection model in which we track the reflected electric field evolution as a function of the number of reflections (Fig. 2e,f) between the LH chiral metamaterial and the metal backplane. As seen in Fig. 2e,f, on multiple reflections, both the reflected $E_{x}$ and $E_{y}$ fields for LCP are diminished (Fig. 2e), while the RCP fields grow (Fig. 2f). This highly asymmetric effect (Fig. 2g,h) comes from the destructive (LCP) and constructive (RCP) interference of the illumination beams and relies on the fact that the planar metamaterial is lossy, anisotropic, and results in linear polarization conversion. More details regarding these requirements, the response of this specific metamaterial, and general design guidelines for planar chiral metamaterials can be found in the Supplementary Information (Supplementary Figs 1-6; Supplementary Notes 1 and 2). It is also important to note that while the handedness of CPL is reversed at the metal backplane the reflected light will see the handedness of the planar chiral metamaterial reversed as well.

Photodetector performance. The high $\mathrm{CD}$ directly leads to enhanced discrimination between LCP and RCP in the CPL photodetectors. To demonstrate this, we fabricated both $\mathrm{LH}$ and RH chiral metamaterial arrays (Fig. 3a,b) on the bottom side of a double-side polished n-type silicon wafer (see the Methods section). The double-side polished wafer is chosen to allow light
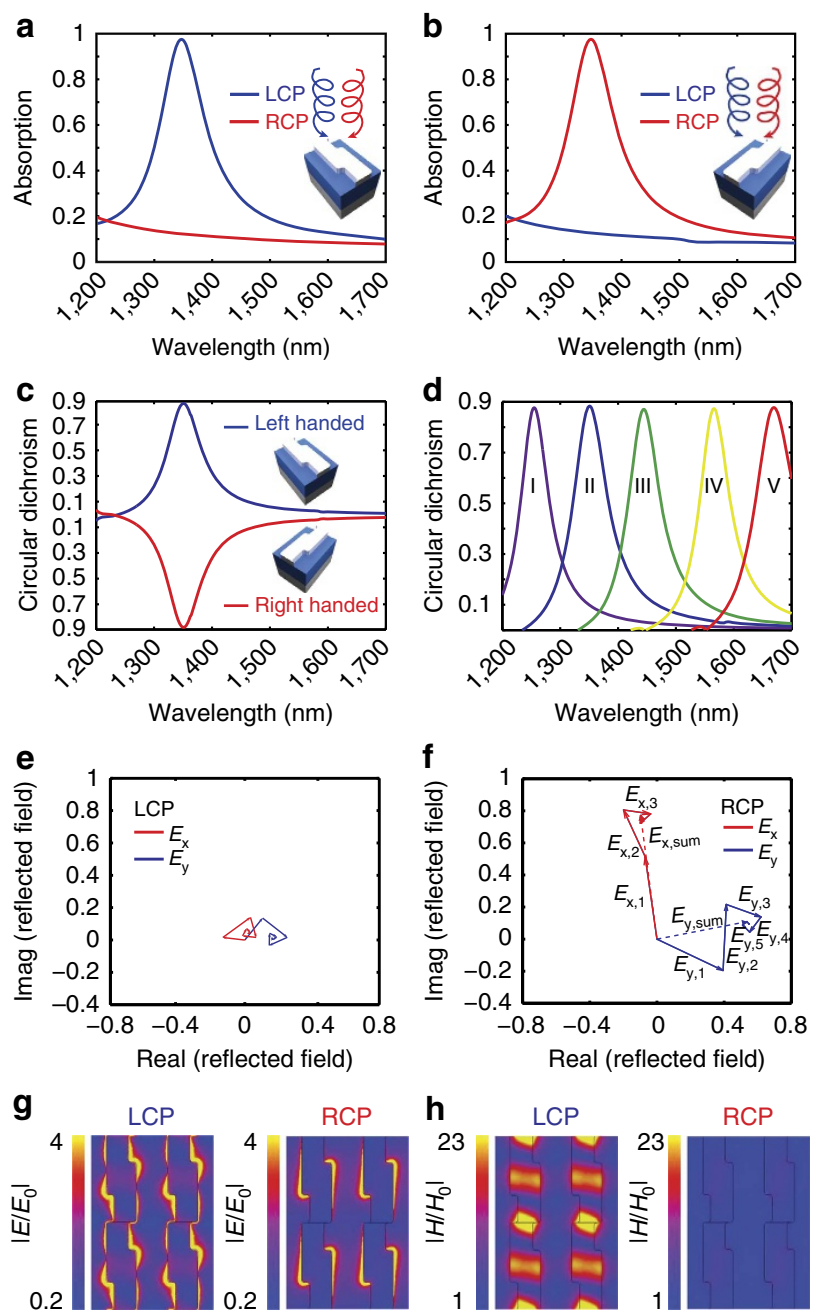

Figure 2 | Simulated optical response of chiral metamaterial.

(a,b) Simulated optical absorption spectra under LCP (blue) and RCP (red) illumination for $\mathrm{LH}$ (a) and RH (b) metamaterials. (c) Circular dichroism spectra for both the $\mathrm{LH}$ (blue) and RH (red) metamaterial. (d) CD as a function of resonator size. Dimensions of the structures $(\mathrm{I}-\mathrm{V})$ are follows: $L_{1}=115,125,130,150$ and $160 \mathrm{~nm} ; L_{2}=95,105,120,130$ and $140 \mathrm{~nm}$; $W_{1}=110,115,120,120$ and $140 \mathrm{~nm} ; W_{2}=85,85,90,90$ and $100 \mathrm{~nm}$; $P_{1}=305,335,370,410$ and $440 \mathrm{~nm} ; P_{2}=230,235,240,240$ and $260 \mathrm{~nm}$, respectively. The other dimensions are the same as Fig. 1a. (e,f) For the $\mathrm{LH}$ metamaterial, the reflected LPL components, $E_{x}$ (red) and $E_{y}$ (blue), on multiple reflections for LCP (e) and RCP (f) light at wavelength of 1,350 nm. $(\mathbf{g}, \mathbf{h})$ Simulated electric $(\mathbf{g})$ and magnetic $(\mathbf{h})$ fields for LCP and RCP illumination at wavelength of $1,350 \mathrm{~nm}$.

illumination from the frontside of the wafer without inducing scattering. A Schottky interface is formed between the chiral plasmonic metamaterial and the semiconductor substrate, allowing us to capture the generated hot electrons. The plasmonic metamaterial was fabricated by first depositing a 1-nm-thick titanium layer (forming the Schottky barrier) followed by a 45-nm-thick aluminum-doped silver film ${ }^{39}$. Aluminum-doped silver was used due to the fact that it helps to reduce the silver film roughness and grain size while also facilitating the formation of an $\mathrm{Al}_{2} \mathrm{O}_{3}$ passivation layer that protects the silver from corrosion $^{39}$.

The experimentally measured optical absorption spectrum of both the LH and RH metamaterial reveals the same absorption bands demonstrated in the simulations. For the LH metamaterial, there is a resonance leading to near unity absorption of LCP light 
a

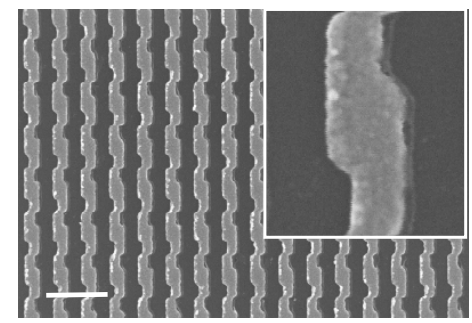

d
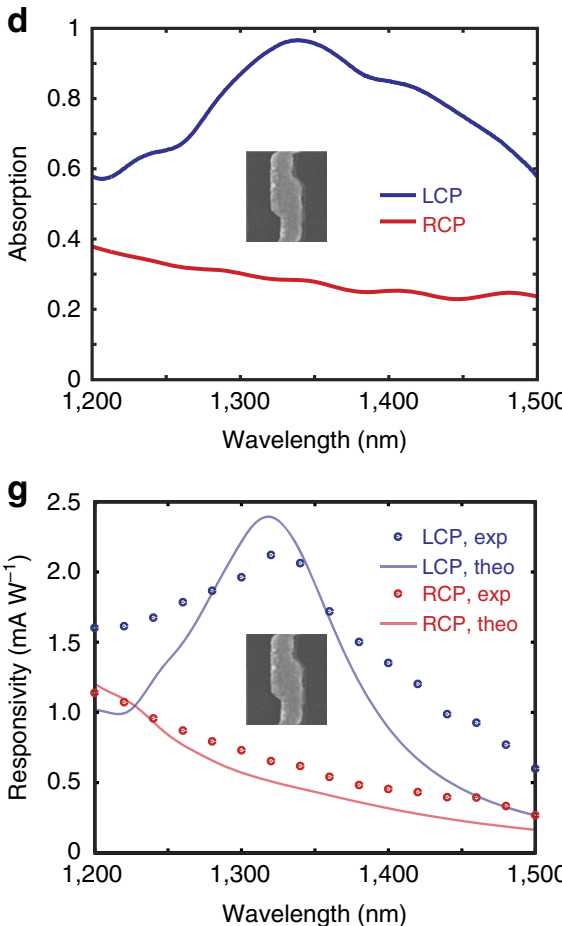

b

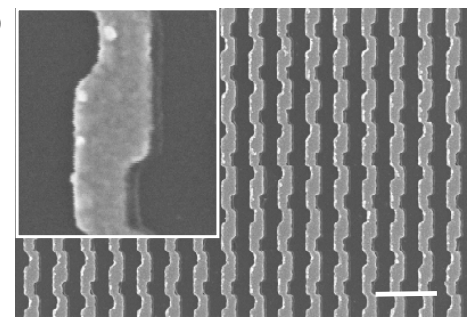

e
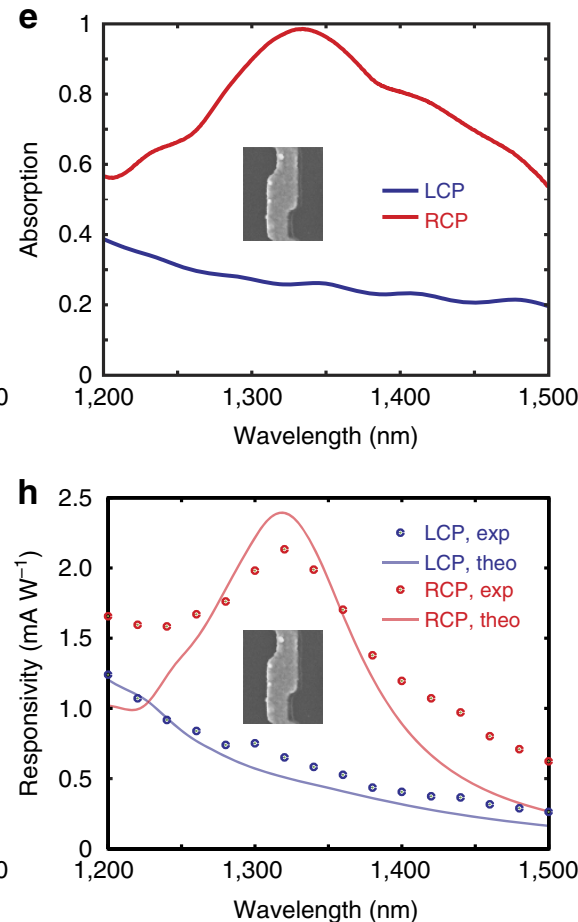

c
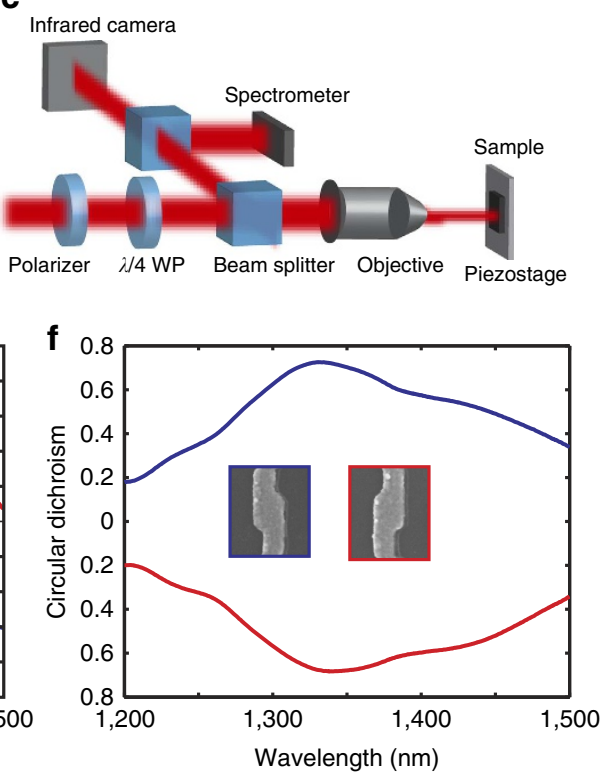

i

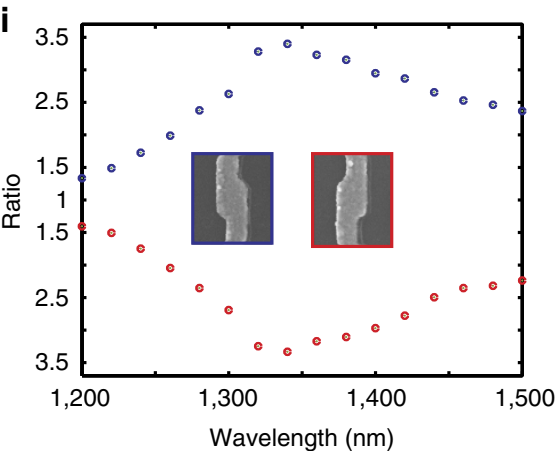

Figure 3 | Experimentally measured optical absorption and photoresponsivity spectra. (a,b) Scanning electron microscope images of the LH (a) and $\mathrm{RH}$ (b) metamaterial before spin coating the PMMA spacer layer. The inset shows a unit cell of the chiral metamaterial. Scale bar, $500 \mathrm{~nm}$. (c) Schematic of experimental set-up. (d,e) Experimentally measured optical absorption spectra under LCP (blue) and RCP (red) illumination for LH (d) and RH (e) metamaterials. (f) Experimentally measured circular dichroism spectra for both LH (blue) and RH (red) metamaterials. (g,h) Experimentally measured (dots) and theoretically calculated (solid curve) photoresponsivity spectra under LCP (blue) and RCP (red) illumination for LH (g) and RH (h) metamaterials. (i) Photocurrent polarization discrimination ratio spectra of $\mathrm{LH}$ and $\mathrm{RH}$ metamaterials. The metamaterials measured have overall areas of $70 \times 70 \mu \mathrm{m}^{2}$.

at $1,340 \mathrm{~nm}$, whereas RCP light is largely reflected (Fig. 3d). The RH metamaterial shows a nearly opposite optical response (Fig. 3e). One will notice that the off-resonant absorption is increased compared with the simulation. This is due to the increased material loss, induced by the aluminum doping of silver and the interfacial 1-nm Ti layer. Even with the slightly increased off-state absorption, the metamaterial still possesses a CD of 0.72 at $1,340 \mathrm{~nm}$ (Fig. 3f), among the highest reported for chiral metamaterials.

Photoresponse spectra of the devices were obtained by illuminating the metamaterials with a circularly polarized laser and measuring photocurrent as a function of the laser handedness and wavelength. The photoresponsivity spectrum (Fig. 3g,h) matches well with the measured absorption spectrum. The peak photoresponsivity of the resonant state reaches $2.2 \mathrm{~mA} \mathrm{~W}^{-1}$, corresponding to a quantum efficiency of $\sim 0.2 \%$ which is comparable to other Schottky diode-based LPL photodetectors working in this wavelength regime ${ }^{32,35}$. This efficiency is two times that of chiral organic semiconductor transistors ${ }^{16}$ and could be further improved by embedding the resonator layer in the silicon ${ }^{31}$. Furthermore, the large $\mathrm{CD}$ translates to a correspondingly large distinction in the photocurrent for LCP and RCP light with a polarization discrimination ratio of 3.4 (Fig. 3i) and a difference in photoresponsivity of $1.5 \mathrm{~mA} \mathrm{~W}^{-1}$, demonstrating the ability to detect and distinguish between left and right hand CPL in an ultracompact detector geometry. It should be noted that the photoresponsivity of this device under non-CPL excitation is about $1.4 \mathrm{~mA} \mathrm{~W}^{-1}$, which is the average value of the resonant and off-resonant polarization state responsivity due to the fact that LPL can be decomposed into two CPL beams with opposite handedness. It is also important to note that although our device responsivity is still low compared with non-chiral InGaAs or Ge detectors $\left(\sim 900 \mathrm{~mA} \mathrm{~W}^{-1}\right)$ in this wavelength range, the later is not able to distinguish CPL. A similar functionality could be achieved by integrating the chiral metamaterial with such semiconductors, however, this does not necessarily produce high polarization selectivity ${ }^{19}$ (Supplementary Fig. 7; Supplementary Note 3) even though a higher quantum efficiency could be realized.

The theoretical photoresponsivity spectra were calculated (solid curve in Fig. 3g,h) based on the creation of energetic electrons due to the collisions with the interface potential wall between the metal and the silicon substrate ${ }^{40,41}$. Local injection current maps were calculated (Supplementary Fig. 8) and the total 
injected current is obtained by integrating over the Schottky interface (see the Methods section). The general trend of the experimental measurements and the theoretically calculated photoresponsivity spectra agrees well, although a resonance broadening is observed in the experimental results. This follows well with the inhomogeneous broadening of the experimentally measured optical absorption spectra compared with simulations (Supplementary Figs 9 and 10), and is most likely due to fabrication imperfections in the metamaterial.

Photocurrent maps were also measured by scanning a focused circularly polarized laser across the array and monitoring photocurrent as a function of spatial position. As can be seen in Fig. 4a, clear distinctions in photocurrent for LCP and RCP light are observed for both $\mathrm{LH}$ and $\mathrm{RH}$ metamaterials. The photocurrent of the chiral metamaterials under the off-resonant polarization is similar to the un-patterned silver bus bar, which confirms the non-resonant behaviour of the structure. The offarray photocurrent is within the noise level of the measurements, which confirms that direct absorption in the silicon is negligible. Furthermore, photocurrent measurements under different laser powers (Supplementary Fig. 11) show good linearity, excluding any non-linear interactions. It also indicates the detector's ability to work over a wide range of incident power, overcoming saturation issues found in chiral organic semiconductors ${ }^{16}$.

As an added benefit, Schottky diode-based photodetectors can provide photocurrent tuning through the application of a sourcedrain bias to the device $e^{30,31,33,34}$ offering flexibility in controlling the polarization discrimination ratio and photoresponsivity. As shown in Fig. 4b, when a negative bias was applied, both the LCP and RCP photocurrent were increased, leading to an increased difference in photoresponsivity. When a positive bias was applied,
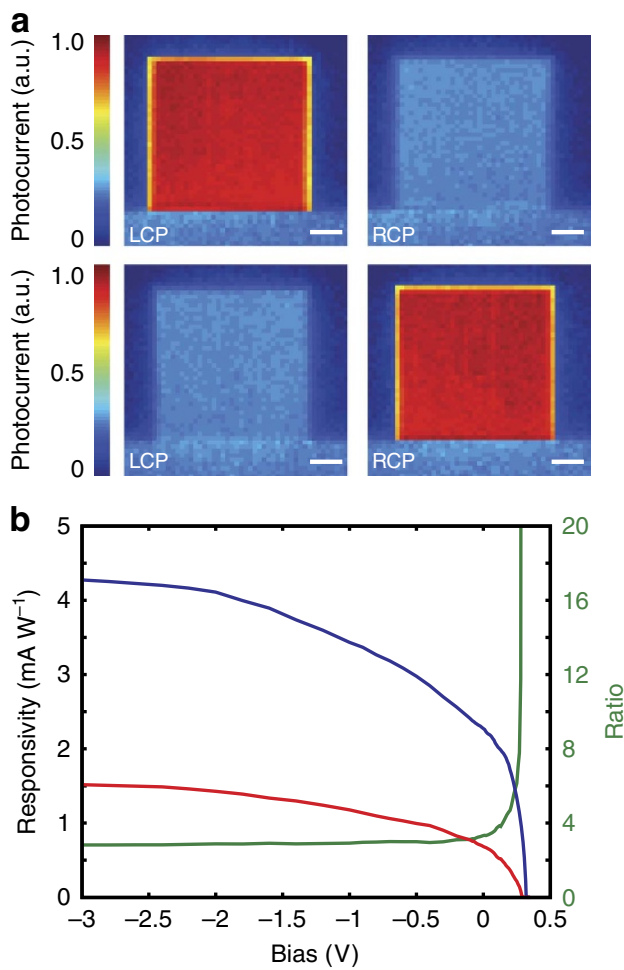

Figure 4 | Spatial scanning and bias-dependent photocurrent.

(a) Scanning photocurrent map of LH (top) and RH (bottom) metamaterials under LCP and RCP illumination. Scale bar, $15 \mu \mathrm{m}$. (b) Bias dependency of photocurrent of a LH metamaterial for LCP (blue) and RCP (red) light under a laser power of $1.5 \mathrm{~mW}$. The green curve shows the polarization discrimination ratio versus bias. despite the decrease in absolute photocurrent, the polarization discrimination ratio or the selectivity can be markedly increased. These measurements indicate how one can generally trade photoresponsivity for a larger polarization discrimination ratio, or vice versa, to meet different application requirements.

Since the chirality of our metamaterial originates from the structure of the antenna layer, instead of an intrinsic material chirality, multiple meta-molecules can be incorporated into a single device to achieve multiple functionalities including CPL, LPL and wavelength selectivity, with only one patterning step. As a proof of concept, we placed LH and RH chiral meta-molecules into a single array to form a spatially non-uniform, pixelated photodetector. The fabricated array is $90 \times 90 \mu \mathrm{m}$, containing roughly 100,621 unit cells. The array was implemented with both $\mathrm{LH}$ and $\mathrm{RH}$ enantiomers that were patterned to create the Vanderbilt University logo (Fig. 5a), where the LH and RH enantiomers fill the black and white regions of the logo, respectively. The shape of our chiral meta-molecules also allows for an electrically connected transition between the oppositehanded enantiomers. As a result, an image of the ' $V$ ' logo, which does not appear under linearly polarized (Fig. $5 b$ ) or un-polarized light, shows a very clear contrast in reflection under LCP and RCP light (Fig. 5c). The performance of this integrated detector was evaluated using scanning photocurrent measurements with focused circularly polarized laser illumination. Good contrast is observed in the photocurrent scanning maps for LCP and RCP (Fig. 5d), indicating the ability to integrate multiple chiral plasmonic meta-molecules into a single-ultracompact device.

\section{Discussion}

In summary, we have demonstrated a CPL detector based on the engineered $\mathrm{CD}$ in plasmonic nanostructures and the hot electron transfer process. This solid-state, hot electron-based chiral metamaterial CPL detector holds the promise of integration, robust and tunable operation ${ }^{29,32,35}$, and a large polarization
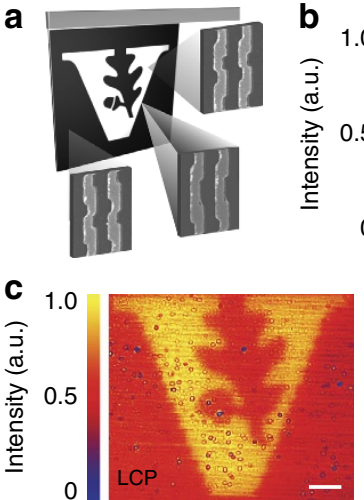

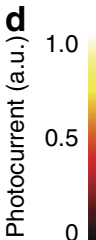
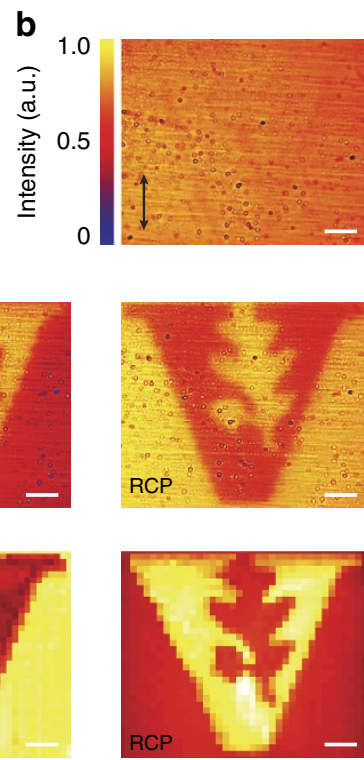

Figure 5 | CPL detector with RH and LH elements patterned into the Vanderbilt University logo. (a) Schematic of the pattern with the LH metamaterial filling the black region and the $\mathrm{RH}$ metamaterial filling the white region. (b) Camera image of the metamaterial under linearly polarized light with polarization along the vertical direction. (c) Camera images of the metamaterial under LCP (left) and RCP (right) illumination. (d) Scanning photocurrent maps of the metamaterial under LCP (left) and RCP (right) illumination. Scale bar, $10 \mu \mathrm{m}$. 
discrimination. Combining our CPL detector with current existing linearly polarized light hot electron photodetectors $^{29,32,34,35}$ could lead to an integrated hot electron polarimeter with the ability to determine the Stokes parameters or the states of polarization of an arbitrarily polarized beam ${ }^{19}$. These CPL detectors could be used in applications ranging from encoded fibre optic and free-space communications to polarimetric imaging, emission and sensing applications using CPL.

\section{Methods}

Simulations. Full-wave electromagnetic simulations were performed using commercially available software (CST Microwave Studio 2013 and Lumerical). In all simulations, periodic boundary conditions were used along the $x$ and $y$ axes and perfectly matched layers were used along the propagation directions. The simulations were performed using an n-type Si half-space above the metamaterial layer followed by the PMMA spacer layer and the silver backplane. The top simulation domain is terminated at silicon such that the reflection from the frontside of the silicon is not taken into account. In the proposed design (Fig. 1a), the optical parameters for Ag were taken from Johnson and Christy ${ }^{42}$ and the damping was increased by three times. In the experimental section, the optical parameters for Al-doped silver and $\mathrm{n}$-type $\mathrm{Si}$ substrate were taken from ellipsometry measurements.

Injection current calculation. In the theoretical treatment, photocurrent arises from the hot electrons that are generated near the metal-semiconductor interface by the plasmonic electric field that is normal to the interface ${ }^{40,41}$. The total injected current from the unit cell is given by

$$
I_{\text {photo-injection }}=C \cdot\left(\hbar \omega-\Delta \phi_{\text {barrier }}\right)^{2} . \int_{\text {injecting surface only }}\left|\mathbf{E}_{\text {normal }}\right|^{2} d s,
$$

where the integral is taken over the metal-Si interface within the unit cell, $C$ is a material constant depending on the Fermi energy and the injecting barrier height, and $\left(\hbar \omega-\Delta \phi_{\text {Barrier }}\right)^{2}$ is a factor reflecting the Fowler law (Supplementary Fig. 10; Supplementary Note 4). The barrier height was measured to be $0.54 \mathrm{eV}$ (Supplementary Fig. 12). The constant $C$ can be estimated from quantum theory and has a characteristic frequency dependence $\sim 1 / \omega^{4}$, which comes from the summation over amplitudes of quantum transitions.

Sample fabrication. The device was fabricated on the bottom side of a double-side polished $<100\rangle$ n-type silicon (Si) wafer $(500-\mu \mathrm{m}$ thick, $1-10 \Omega \mathrm{cm})$. After spin coating the substrate with 130-nm-thick PMMA, the antenna arrays were defined using electron beam lithography. The LH and RH arrays had an overall area of $70 \times 70 \mu \mathrm{m}^{2}$ and the VU Logo array was $90 \times 90 \mu \mathrm{m}^{2}$. After cold development in methyl isobutyl ketone (MIBK):isopropyl alcohol (IPA) 1:3, the sample was cleaned with an IPA rinse and dried under a $\mathrm{N}_{2}$ stream. The sample was immersed in 10:1 buffered oxide etchant for $10 \mathrm{~s}$ to completely remove the native oxide from the Si. It was then immediately transferred into an evaporation chamber followed by evaporation of $1 \mathrm{~nm} \mathrm{Ti}$ and co-deposition of a 45-nm-thick Al-doped silver film. The interfacial Ti layer produces a Schottky barrier height of $\phi_{\mathrm{B}}=0.54 \mathrm{eV}$. The silver deposition rate was $2 \AA \mathrm{s}^{-1}$ and the aluminum deposition rate was $0.2 \AA \mathrm{s}^{-1}$, leading to an aluminum concentration of $\sim 9 \%$. After the co-deposition a lift-off process was used to yield the structures shown in Fig. 3a,b. The obtained metamolecule dimensions are $L_{1}=130 \mathrm{~nm}, L_{2}=110 \mathrm{~nm}, W_{1}=110 \mathrm{~nm}, W_{2}=80 \mathrm{~nm}$, $P_{1}=350 \mathrm{~nm}$ and $P_{2}=230 \mathrm{~nm}$. Finally, a 160 -nm-thick PMMA spacer layer was created by spin coating and followed by thermal evaporation of a $100-\mathrm{nm}$-thick silver backplane.

Characterization. The optical absorption and CD spectrum were obtained using a broadband white-light source and a custom infrared microscope coupled with a grating spectrometer (Horiba, iHR320). CPL was generated with a polarizer combined with a quarter waveplate. The quality of the CPL was checked with an additional polarizer. To measure the reflection spectra, a silver mirror was used as the reference. The reflection from the frontside of the wafer, measured using an un-patterned single-side polished silicon wafer with same doping level, is subtracted from the reflection from the patterned sample. This matches the configuration in the simulations. The transmission is zero due to the optically thick silver backplane. An Ohmic contact on the n-type silicon substrate was achieved by indium soldering. The sample was wire bonded to a chip carrier to perform the photocurrent measurements. The photocurrent measurements were obtained using a custom scanning photocurrent microscope along with a tunable laser (Coherent Mira OPO), digital source meter (Keithley 2400), lock-in amplifier (SR830) and pre-amplifier (DL Instruments 1211). The laser was mechanically chopped (Thorlabs MC2000 \& MC1F10) at $950 \mathrm{~Hz}$ with a lock-in integration time of $300 \mathrm{~ms}$. Photocurrent maps were generated by raster scanning a focused circularly polarized laser ( $\sim 2 \mu \mathrm{m}$ full-width at half-maximum) across the array and monitoring photocurrent as a function of spatial position. The scan step size is
$2 \mu \mathrm{m}$ in the $x$ and $y$ directions and for each measurement we dwell $1 \mathrm{~s}$ to stabilize the piezoelectric stage before recording data.

\section{References}

1. Sherson, J. F. et al. Quantum teleportation between light and matter. Nature 443, 557-560 (2006).

2. Wagenknecht, C. et al. Experimental demonstration of a heralded entanglement source. Nat. Photon. 4, 549-552 (2010).

3. Togan, E. et al. Quantum entanglement between an optical photon and a solid-state spin qubit. Nature 466, 730-734 (2010).

4. Farshchi, R., Ramsteiner, M., Herfort, J., Tahraoui, A. \& Grahn, H. T. Optical communication of spin information between light emitting diodes. Appl. Phys. Lett. 98, 162508 (2011).

5. Greenfield, N. J. Using circular dichroism spectra to estimate protein secondary structure. Nat. Protoc. 1, 2876-2890 (2006).

6. Stanciu, C. D. et al. All-optical magnetic recording with circularly polarized light. Phys. Rev. Lett. 99, 047601 (2007).

7. Feynman, R. P., Leighton, R. B. \& Sands, M. The Feynman Lectures on Physics (Addison Wsley Longman, 1970).

8. Chiou, T.-H. et al. Circular polarization vision in a stomatopod crustacean. Curr. Biol. 18, 429-434 (2008).

9. Zhang, Y. J., Oka, T., Suzuki, R., Ye, J. T. \& Iwasa, Y. Electrically switchable chiral light-emitting transistor. Science 344, 725-728 (2014).

10. Zhang, S. et al. Chiral surface plasmon polaritons on metallic nanowires. Phys. Rev. Lett. 107, 096801 (2011).

11. Yu, N. et al. A broadband, background-free quarter-wave plate based on plasmonic metasurfaces. Nano Lett. 12, 6328-6333 (2012).

12. Gansel, J. K. et al. Gold helix photonic metamaterial as broadband circular polarizer. Science 325, 1513-1515 (2009).

13. Turner, M. D. et al. Miniature chiral beamsplitter based on gyroid photonic crystals. Nat. Photon. 7, 801-805 (2013).

14. Rodríguez-Fortuño, F. J., Barber-Sanz, I., Puerto, D., Griol, A. \& Martínez, A. Resolving light handedness with an on-chip silicon microdisk. ACS Photon. 1, 762-767 (2014).

15. Sharma, V., Crne, M., Park, J. O. \& Srinivasarao, M. Structural origin of circularly polarized iridescence in jeweled beetles. Science 325, 449-451 (2009).

16. Yang, Y., da Costa, R. C., Fuchter, M. J. \& Campbell, A. J. Circularly polarized light detection by a chiral organic semiconductor transistor. Nat. Photon. 7, 634-638 (2013).

17. Valev, V. K., Baumberg, J. J., Sibilia, C. \& Verbiest, T. Chirality and chiroptical effects in plasmonic nanostructures: fundamentals, recent progress, and outlook. Adv. Mater. 25, 2517-2534 (2013).

18. Ben-Moshe, A., Maoz, B. M., Govorov, A. O. \& Markovich, G. Chirality and chiroptical effects in inorganic nanocrystal systems with plasmon and exciton resonances. Chem. Soc. Rev. 42, 7028-7041 (2013).

19. Afshinmanesh, F., White, J. S., Cai, W. \& Brongersma, M. L. Measurement of the polarization state of light using an integrated plasmonic polarimeter. Nanophotonics 1, 125-129 (2012).

20. Fedotov, V. A., Schwanecke, A. S., Zheludev, N. I., Khardikov, V. V. \& Prosvirnin, S. L. Asymmetric transmission of light and enantiomerically sensitive plasmon resonance in planar chiral nanostructures. Nano Lett. 7, 1996-1999 (2007).

21. Kuzyk, A. et al. DNA-based self-assembly of chiral plasmonic nanostructures with tailored optical response. Nature 483, 311-314 (2012)

22. Schäferling, M., Yin, X., Engheta, N. \& Giessen, H. Helical plasmonic nanostructures as prototypical chiral near-field sources. ACS Photon. 1, 530-537 (2014).

23. Hopkins, B., Poddubny, A. N., Miroshnichenko, A. E. \& Kivshar, Y. S. Circular dichroism induced by Fano resonances in planar chiral oligomers. Preprint at http://arxiv.org/abs/1412.1120 (2014).

24. Zhao, Y., Belkin, M. A. \& Alù, A. Twisted optical metamaterials for planarized ultrathin broadband circular polarizers. Nat. Commun. 3, 870 (2012).

25. Cui, Y., Kang, L., Lan, S., Rodrigues, S. \& Cai, W. Giant chiral optical response from a twisted-arc metamaterial. Nano Lett. 14, 1021-1025 (2014).

26. Ben-Moshe, A. et al. Enantioselective control of lattice and shape chirality in inorganic nanostructures using chiral biomolecules. Nat. Commun. 5, 4302 (2014).

27. Clavero, C. Plasmon-induced hot-electron generation at nanoparticle/metaloxide interfaces for photovoltaic and photocatalytic devices. Nat. Photon. 8, 95-103 (2014).

28. Brongersma, M. L., Halas, N. J. \& Nordlander, P. Plasmon-induced hot carrier science and technology. Nat. Nanotechnol. 10, 25-34 (2015).

29. Knight, M. W., Sobhani, H., Nordlander, P. \& Halas, N. J. Photodetection with active optical antennas. Science 332, 702-704 (2011).

30. Goykhman, I., Desiatov, B., Khurgin, J., Shappir, J. \& Levy, U. Waveguide based compact silicon Schottky photodetector with enhanced responsivity in the telecom spectral band. Opt. Express 20, 28594-28602 (2012). 
31. Knight, M. W. et al. Embedding plasmonic nanostructure diodes enhances hot electron emission. Nano Lett. 13, 1687-1692 (2013).

32. Sobhani, A. et al. Narrowband photodetection in the near-infrared with a plasmon-induced hot electron device. Nat. Commun. 4, 1643 (2013).

33. Wang, F. \& Melosh, N. A. Power-independent wavelength determination by hot carrier collection in metal-insulator-metal devices. Nat. Commun. 4, 1711 (2013).

34. Chalabi, H., Schoen, D. \& Brongersma, M. L. Hot-electron photodetection with a plasmonic nanostripe antenna. Nano Lett. 14, 1374-1380 (2014).

35. Li, W. \& Valentine, J. Metamaterial perfect absorber based hot electron photodetection. Nano Lett. 14, 3510-3514 (2014).

36. Linic, S., Christopher, P. \& Ingram, D. B. Plasmonic-metal nanostructures for efficient conversion of solar to chemical energy. Nat. Mater. 10, 911-921 (2011).

37. Mubeen, S. et al. An autonomous photosynthetic device in which all charge carriers derive from surface plasmons. Nat. Nanotechnol. 8, 247-251 (2013).

38. Giugni, A. et al. Hot-electron nanoscopy using adiabatic compression of surface plasmons. Nat. Nanotechnol. 8, 845-852 (2013).

39. Gu, D., Zhang, C., Wu, Y.-K. \& Guo, L. J. Ultrasmooth and thermally stable silver-based thin films with subnanometer roughness by aluminum doping. ACS Nano 8, 10343-10351 (2014).

40. Govorov, A. O., Zhang, H. \& Gun'ko, Y. K. Theory of photoinjection of hot plasmonic carriers from metal nanostructures into semiconductors and surface molecules. J. Phys. Chem. C 117, 16616-16631 (2013).

41. Govorov, A. O. \& Zhang, H. Kinetic density functional theory for plasmonic nanostructures: breaking of the plasmon peak in the quantum regime and generation of hot electrons. J. Phys. Chem. C 119, 6181-6194 (2015)

42. Johnson, P. B. \& Christy, R. W. Optical constants of the noble metals. Phys. Rev. B 6, 4370-4379 (1972).

\section{Acknowledgements}

W.L. and J.V. were supported by the National Science Foundation (NSF) under program CBET-1336455. Z.C. was supported by a National Science Foundation Graduate
Research Fellowship. W.W. was supported by the Office of Naval Research under grant number N00014-14-1-0475. L.V. and A.O.G. were supported by the US Army Research Office under grant number W911NF-12-1-0407 and by the Volkswagen Foundation (Germany). Device fabrication was performed at the Vanderbilt Institute of Nanoscale Science and Engineering, we thank the staff for their support.

\section{Author contributions}

W.L. and J.V. conceived the concept. W.L. designed the metamaterial and conducted numerical simulations. W.L. and Z.J.C. performed the device fabrication and experimental characterization. L.V. and A.O.G. performed theoretical injection current calculations and assisted in simulations. W.W performed the multiple reflection calculations. J.V. supervised the project. All authors discussed the results and contributed to writing the manuscript.

\section{Additional information}

Supplementary Information accompanies this paper at http://www.nature.com/ naturecommunications

Competing financial interests: The authors declare no competing financial interests.

Reprints and permission information is available online at http://npg.nature.com/ reprintsandpermissions/

How to cite this article: Li, W. et al. Circularly polarized light detection with hot electrons in chiral plasmonic metamaterials. Nat. Commun. 6:8379 doi: 10.1038/ncomms9379 (2015).

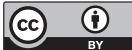

This work is licensed under a Creative Commons Attribution 4.0 International License. The images or other third party material in this article are included in the article's Creative Commons license, unless indicated otherwise in the credit line; if the material is not included under the Creative Commons license, users will need to obtain permission from the license holder to reproduce the material To view a copy of this license, visit http://creativecommons.org/licenses/by/4.0/ 